\title{
Designing resilient networks using multicriteria metaheuristics
}

\author{
Crina Grosan • Ajith Abraham • Aboul Ella Hassainen
}

(C) Springer Science+Business Media, LLC 2008

\begin{abstract}
The paper deals with the design of resilient networks that are fault tolerant against link failures. Usually, fault tolerance is achieved by providing backup paths, which are used in case of an edge failure on a primary path. We consider this task as a multiobjective optimization problem: to provide resilience in networks while minimizing the cost subject to capacity constraint. We propose a stochastic approach, which can generate multiple Pareto solutions in a single run. The feasibility of the proposed method is illustrated by considering several network design problems using a single weighted average of objectives and a direct multiobjective optimization approach using the Pareto dominance concept.
\end{abstract}

Keywords Resilient networks · Multiobjective optimization $\cdot$ Metaheuristics $\cdot$ Pareto solutions

\section{Introduction}

Reliability has been a traditional goal within telecommunication network design. There has been some recent research

C. Grosan ( $\square)$

Department of Computer Science, Babes-Bolyai University, Cluj-Napoca, Romania

e-mail: cgrosan@cs.ubbcluj.ro

\section{A. Abraham}

Centre for Quantifiable Quality of Service, Centre of Excellence

Norwegian University of Science and Technology, Trondheim,

Norway

e-mail: ajith.abraham@ieee.org

\section{A.E. Hassainen}

College of Business Administration, Quantitative Methods and IS

Department, Kuwait University, P.O. Box 5486, Safat 13055,

Kuwait

e-mail: abo@cba.edu.kw interest in developing algorithms for problems of guaranteeing resilience against failures. Due to the fact that the assurance of continuity in traffic is a vital demand in today's networks, we have to be able to provide backup paths at the moment a failure on a edge (or in multiple edges) on the primary path occurs. For this, the backup path has to be built a priory.

There are several attempts to solve the resilience and path allocation problem in different ways. Chekuri et al. [1] deal with edge failure by building simultaneous primary and backup paths but they are only considering the case of uncapacitated networks. A hybrid genetic algorithm which deals with alternative backup paths was proposed by Chekuri et al. [1], but the problem is not considered as multiobjective. A multiobjective network design approach is proposed by Banarjee and Kumar [3] and by Yeh [11], but not directly treating resilience. Being an important and interesting research topic, in the literature, there is a h0uge amount of work related to network resilience [2, 4-10, 12, 24] are some of them. Some surveys on resilient network technology, planning and optimization can be found in [13-15].

De Vleeschouwer and Frossard [20] investigated methods for efficient packet loss recovery by retransmission, and build on explicit congestion control mechanisms to decouple the packet loss detection from the congestion feedback signals. They proposed a novel retransmission timer to deal with multiple losses of data segments and, in consequence, to allow for aggressive reset of the connection recovery timer. For different retransmission strategies that respectively rely on conventional cumulative acknowledgments or accurate loss monitoring, authors illustrated how the principles underlying the TCP retransmission mechanisms have to be adapted in order to take advantage of an explicit congestion feedback. 
Wouhaybi and Campbell [21] designed and evaluated a peer-to-peer algorithm for building resilient low-diameter peer-to-peer topologies that can resist different types of organized and targeted malicious behavior. The proposed framework leverages the strengths of some of the existing approaches without inheriting their weaknesses and is capable of building topologies of nodes that follow a powerlaw while being fully distributed requiring no central server, thus, eliminating the possibility of a single point of failure in the system.

Antonino et al. [22] presented the architecture of a Wavelength Division Multiplexing (WDM) optical packet network which is targeted to assess the effectiveness of optical technologies with respect to electronic ones, trying to identify an optimal mix of the two technologies. The developed architecture shows interesting resilience properties that enable the design of fast fault-recovery schemes.

The explosive growth of data generation has invoked a big challenge to design a resilient and scalable interconnection network which consolidates hundreds even thousands of storage nodes to satisfy both the bandwidth and storage capacity requirements. Deng [23] proposed a resilient interconnection network for storage cluster systems by employing multiple spare links between any two storage nodes to offer strong resilience to reduce the impact of the failures of links, switches, and storage nodes. A metric named resilience coefficient is also proposed to measure the interconnection network.

In this paper, we focus on the simultaneous allocation of primary and backup paths. We also formulate the problem as dealing with multiple criteria in the same time where several objectives can be formulated. In this research, we deal with three criteria: (1) minimize the network cost, (2) minimize the number of common links between primary and its corresponding backup path (so that in case an edge (link) failure occurs on the primary path the chances that the same edge belongs to the backup path also and makes the traffic impossible to continue is minimal) and (3) maximize the number of common links between all the backup paths (in the situation that there are several source-destination flows to be fulfilled at the same time). We also take into consideration that an edge failure can affect only one of the primary paths at one time and there is no requirement for using both backup paths as reserve in the same time. One approach to planning such networks is by simulation, where a stochastic optimization technique is applied.

We provide a detailed description of the algorithm proposed in the following section. The ways in which a solution is initialized and improved are widely described and an illustrative example is presented in Sect. 2. In Sect. 3 the proposed approach is illustrated followed by several experimental results in Sect. 4. Conclusions are provided towards the end.

\section{Problem formulation}

Since in today's networks, due to the demand's importance we cannot simply rely on a single network path, we have to a priori build a path, which can be used to reroute the traffic in case a failure occurs on the primary path. It is necessary to predefine a backup path (or backup sub-paths) if:

- the recovery of full capacity after failure shall be guaranteed;

- the recovery shall be almost immediate $(\sim 50 \mathrm{~ms})$, i.e. no noticeable service reduction.

Recovery by predefining backup paths is referred to as "protection". Recovery by finding back-up paths when (after) a failure has occurred is referred to as "restoration" [13, 15]. This paper focuses on the cases where it is guaranteed that no more than a fixed number of edge-failures could occur.

We then consider the problem of simultaneous primary and backup path allocation. We are given specifications of the traffic to be handled, and to make the provision for both primary as well as the backup networks. On the arrival of a pair (source, destination), the task is to find both a primary path and a backup path between them.

\subsection{Network parameters}

We consider the following network parameters:

- A bi-directed graph $G=(V, E)$;

$\circ$ the adjacency matrix;

- the capacity of each link: cap : $E \rightarrow R^{+}$;

- the cost associated to each link: $\operatorname{co}: E \rightarrow R^{+}$;

- a pair source-destination $(s, d) \in V$ (or a set of pairs $\left(s_{i}, d_{i}\right), i=1, \ldots, N, N, \geq 1$, in the general case) and the requirements.

\subsection{Objective functions}

The goal is to find a minimal (cost wise) path between these nodes as well as to assure that the capacity of the edges is not overloaded, which is formulated in the objectives considered below.

Objective 1 Minimize the total cost of the paths (primary and backup) between source and destination:

$$
\begin{gathered}
\operatorname{minimize} \sum_{i=1}^{N} \operatorname{cost}\left(\operatorname{primary} \operatorname{path}\left(s_{i}, d_{i}\right)\right) \\
+\sum_{i=1}^{N} \operatorname{cost}\left(\operatorname{backup} \operatorname{path}\left(s_{i}, d_{i}\right)\right)
\end{gathered}
$$


Objective 2 Suppose an edge fails; in order to ensure the network survivability we have to use the backup path. But for this we have to ensure that the failed edge is not also a part of the backup path. This is formulated as a simple objective (criterion) in our model: minimize the number of common edges between primary and backup paths:

$$
\begin{array}{r}
\operatorname{minimize} \sum_{i=1}^{N} \text { no of common edges(primary } \operatorname{path}\left(s_{i}, d_{i}\right), \\
\left.\operatorname{backup} \operatorname{path}\left(s_{i}, d_{i}\right)\right) .
\end{array}
$$

Objective 3 This objective is used while dealing with shared backup path protection (the objective requires that the backup paths (in the situation in which we have multiple source-destination pairs) should share as many edges as possible):

$$
\begin{array}{r}
\operatorname{maximize} \sum_{\substack{i=1, j=1, i \neq j}}^{N} \text { no of common edges(backup path }\left(s_{i}, d_{i}\right), \\
\left.\operatorname{backup} \operatorname{path}\left(s_{j}, d_{j}\right)\right) .
\end{array}
$$

\subsection{Constraints}

Capacity is treated as a constraint. Once a solution is generated or obtained by modifying an existing one (as explained in the following sections) its validity is verified. This validity refers to the capacity restriction. If capacity is overloaded in at least one link, then the solution is not taken into consideration.

As evident, the problem involves the simultaneous optimization of multiple objectives. There are several ways to deal with a multiobjective optimization problem. Two of the standard approaches are weighted sum methodwhich combines the objectives and reduces the problem to a single objective optimization one- and Pareto dominance based method-which treats the problem as multiobjective and uses the standard Pareto dominance relationship.

Definition 1 (Pareto dominance) Consider a maximization problem. Let $x, y$ be two decision vectors (solutions) from the definition domain.

Solution $x$ dominates $y$ (also written as $x \succ y$ ) if and only if the following conditions are fulfilled:

(i) $f i(x) \geq f i(y) ; \forall i=1,2, \ldots, n$;

(ii) $\exists j \in\{1,2, \ldots, n\}: f j(x)>f j(y)$.

That is, a feasible vector $x$ is Pareto optimal if no feasible vector $y$ can increase some criterion without causing a simultaneous decrease in at least one other criterion [18, 19].

The weighted-sum method is a traditional, popular method that parametrically changes the weights among objective functions to obtain the Pareto front $[16,17]$.

Let us consider we have the objective functions $f_{1}, f_{2}$, $\ldots, f_{n}$. This method takes each objective function and multiplies it by a fraction of one, the "weighting coefficient" which is represented by $w_{i}$. The modified functions are then added together to obtain a single cost function, which can easily be solved using any method which can be applied for single objective optimization.

Mathematically, the new function is written as:

$\sum_{i=1}^{n} w_{i} f_{i}$

where $0 \leq w i \leq 1$ and $\sum_{i=1}^{n} w_{i}=1$.

\section{Description of the proposed multiobjective optimization approach}

In this section, we describe the way in which a solution is build and further improved. The model can be used by any of the approaches mentioned above (weighted sum method and Pareto dominance model). In the proposed model, a solution represents all the required paths to be designed together with their corresponding backup paths. For example, if there are 2 source nodes and 2 destination nodes, the task is to find 4 primary paths between sources and destinations as well as 4 backup paths. The developed solution represents all the 8 paths. If we have just one source node and one destination node then the solution will only consists of two paths (the primary and the backup).

In order to build a solution, we generate a set of valid paths between each source and each destination node required. From each set, the primary and backup paths are selected which will compose the solution. This selection is done on a random basis. A set of such feasible solutions is generated and maintained during the search process. Improvement techniques are used in order to assure a convergence to a better solution.

\subsection{Solution design}

In order to build a path, following are the steps:

Step 1. Start with the source node as current node.

Step 2. If there is a direct path (link) from the current node to the destination node, then move to destination node. Otherwise randomly chose another node from the network, which is connected to the current node and set it as new current node. 
Step 3. If the current node is the desired destination node then stop. Otherwise go to Step 2.

The following constraints are taken into account:

- each node can be used at most one time in a path (for avoiding cycles);

- if a node is arrived such that further movement to another node (stuck there) is impossible (because all the connected nodes were previously used), then that solution is aborted.

\subsection{Solution improvement}

There are two ways to improve a solution:

By applying some improvements directly to that solution or by combining 2 (or more) solutions in order to get better ones and to obtain some diversity.

For the first case, a solution can be improved in one of the following ways:

1. Rebuild and existing path (primary or backup) from a given node.

2. Replace an existing path with another one from the set of paths generated in the beginning of the search process.

For the second case, the two solutions which are to be combined need to have a common node different from the first and second one. Both these techniques for improving solutions are considered.

\subsubsection{First improvement technique}

In order to improve a solution designed using the above procedure, some modifications on the existing paths are performed as follows:

- one primary path is randomly selected from the ones initially designed. One node on this path is also randomly generated. From the selected node, the path is re-built by considering another path to reach the destination different from the current one.

- one backup path is randomly selected from the ones initially generated and a procedure similar to the one used for the primary path is applied.

\subsubsection{Second improvement technique}

Another way in which a solution can be improved consists on exchanging a whole path with another one from the existing set (initially generated) of paths. One path (either primary or backup) between each source and destination is picked at one time and exchanged with another one. The newly obtained solution is again compared with the initial solution and the rules described above are followed. The improving procedures are applied for each of the solutions.
These improving steps are repeated for a successive number of iterations. At the end of these iterations, the nondominated solutions among all the obtained solutions are considered.

The newly obtained solution is compared to the previous solution (which was modified). In order to choose between two solutions and compare them, Pareto dominance relationship is used:

- If it dominates the previous solution then the new solution is kept;

- If it is dominated by the previous solution then the previous solution is kept;

- If the initial solution and the new obtained solution are nondominated then one of them (randomly chosen between the two) is kept.

\subsubsection{Third improvement technique}

Two paths from two different solutions (corresponding to the same primary or backup path) can be combined if they have a common node different from the first and last one (representing source and destination). Their combination is similar to a cutting-point procedure and consist of exchanging the sub-paths starting with the common node.

For example, if we have the paths:

$2-7-9-4-3-8-1-5$

$2-8-11-9-6-5$

It is noticed that there are 2 common nodes, 9 and 8 . Then, a possible combination of the 2 paths gives the following two new paths:

$2-7-9-6-5$

$2-8-11-9-4-3-8-1-5$

A correction is then applied, which basically consists of removing all the cycles from a path. For instance, from the second path we can remove the cycle 811943 and the new path will be 2815 . In the case that there are multiple cycles starting and ending in the same node, we will keep the shortest one.

The pseudo code of the proposed approach is summarized as follows:

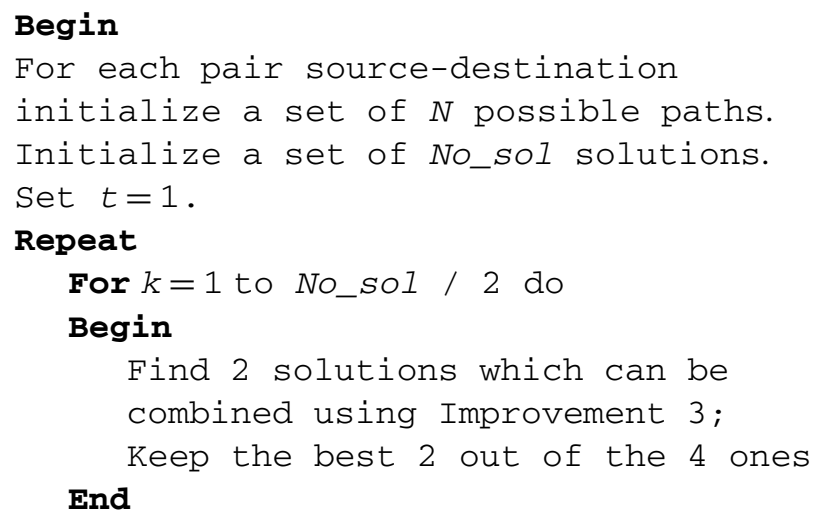




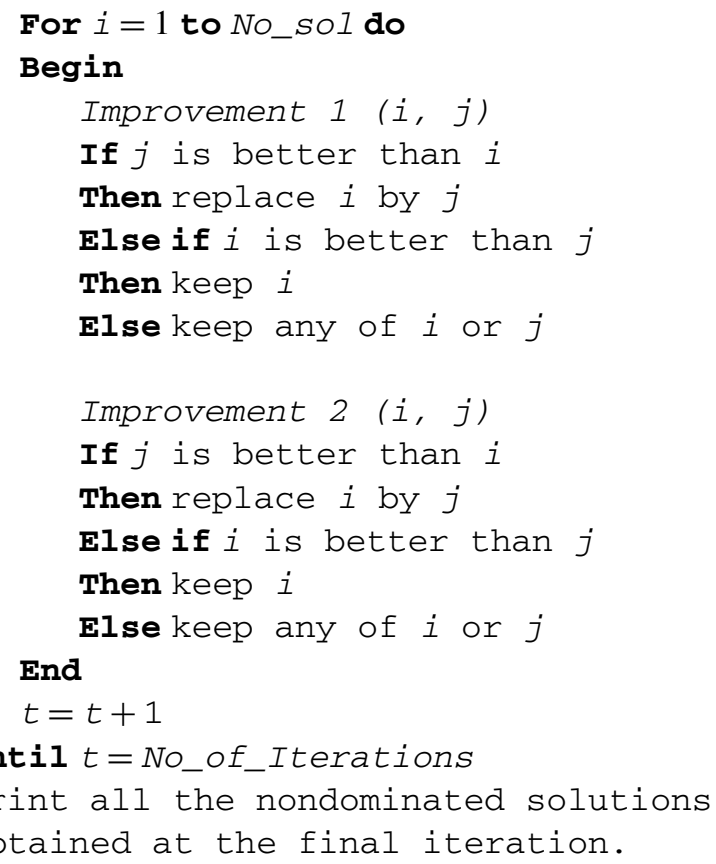

End.

\section{Remark}

(i) The Improvements 1 and 2 procedures refer to the solution obtained by applying that specific improvement.

(ii) No_of_iterations is a priori known and represents the stop criterion.

\section{Experiments and discussions}

In order to emphasize the performance of the proposed approach we considered three examples. For the first example, the whole procedure is illustrated in detail so that the reader can easily follow all the explanations about the proposed technique in the previous section. The second example is the well known Europe network benchmark having 37 nodes and 55 connections. The third example consists of 65 nodes and 108 connections.

\subsection{Experimental example 1}

We consider the network given in Fig. 1. The cost associated to each pair of connected nodes is considered to be one. We have two source nodes (node 1 and node 5) and two destination nodes (node 8 and node 13). The goal is to efficiently design two primary paths and two corresponding backup paths.

Solution initialization The task is to determine 4 paths (two primary paths and two backup paths). First, a set of paths between node 1 and node 5 and a similar set of paths between node 8 and node 13 are generated and then some paths are picked randomly from these sets to construct the initial set of solutions. Then, an initialization of these sets (having 10 elements) would appear as follows:

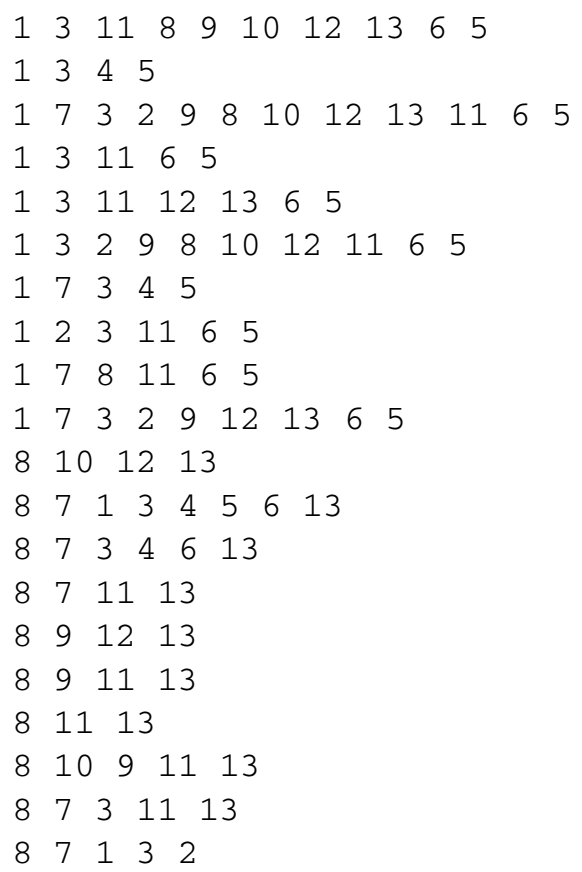

6 of the formulated solutions are given below (P and B refer to primary and backup paths respectively):

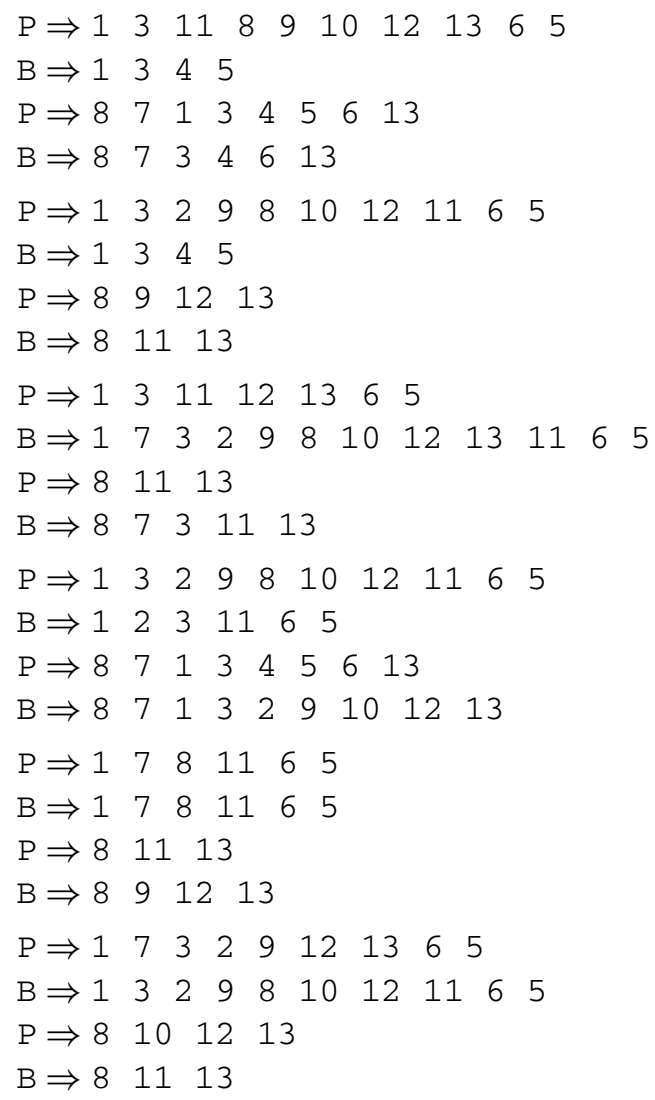




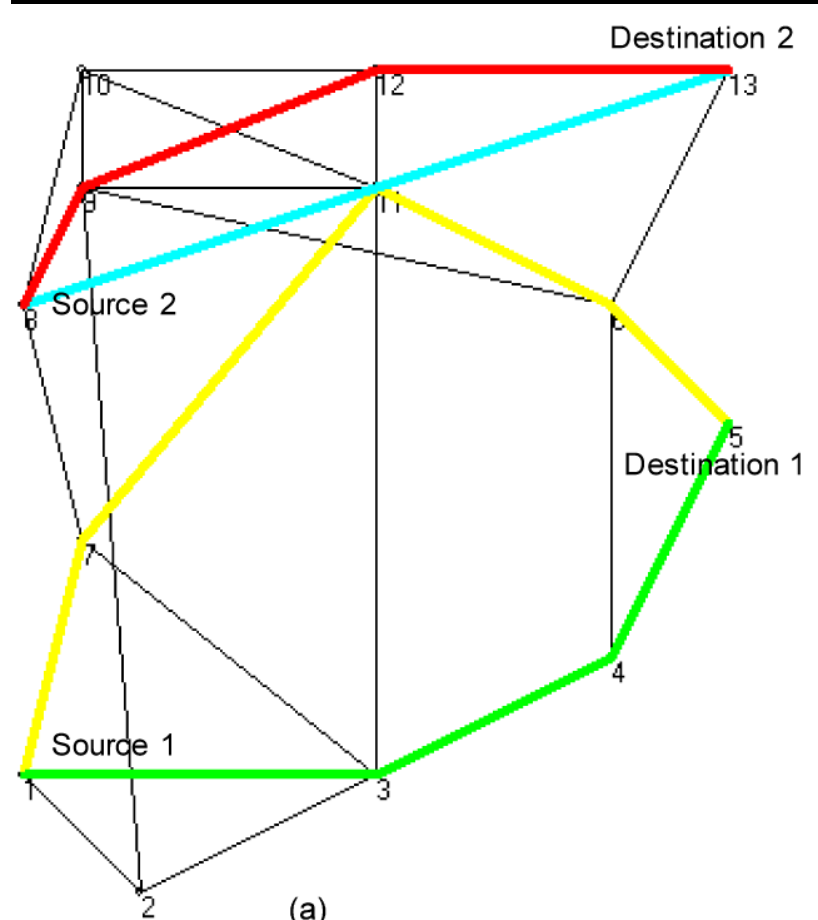

(a)

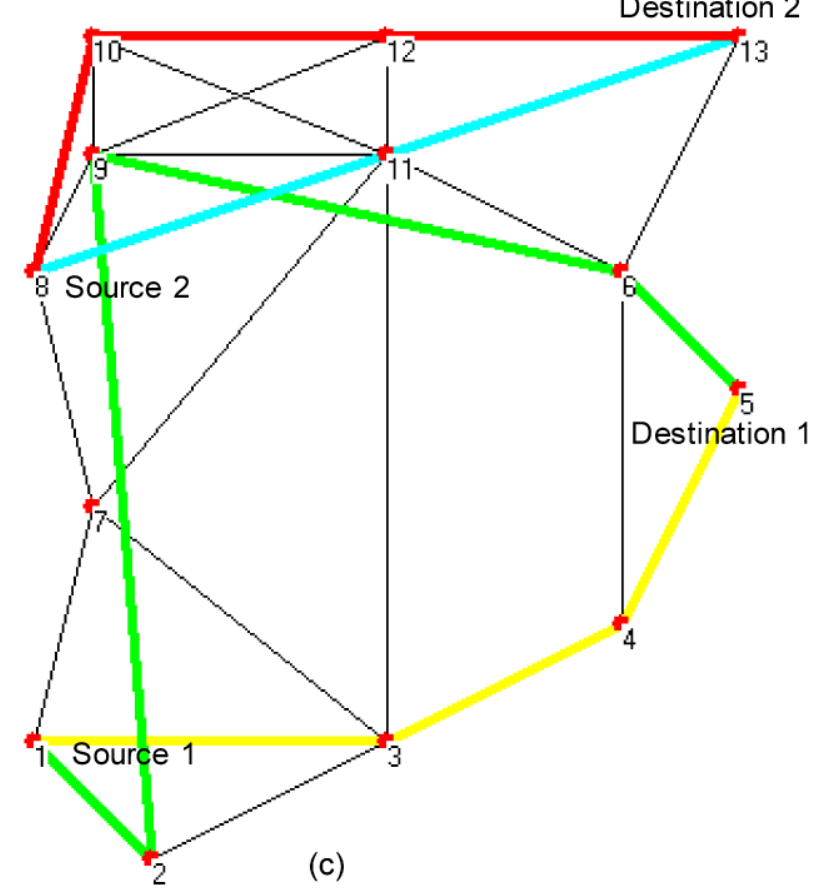

Destination 2

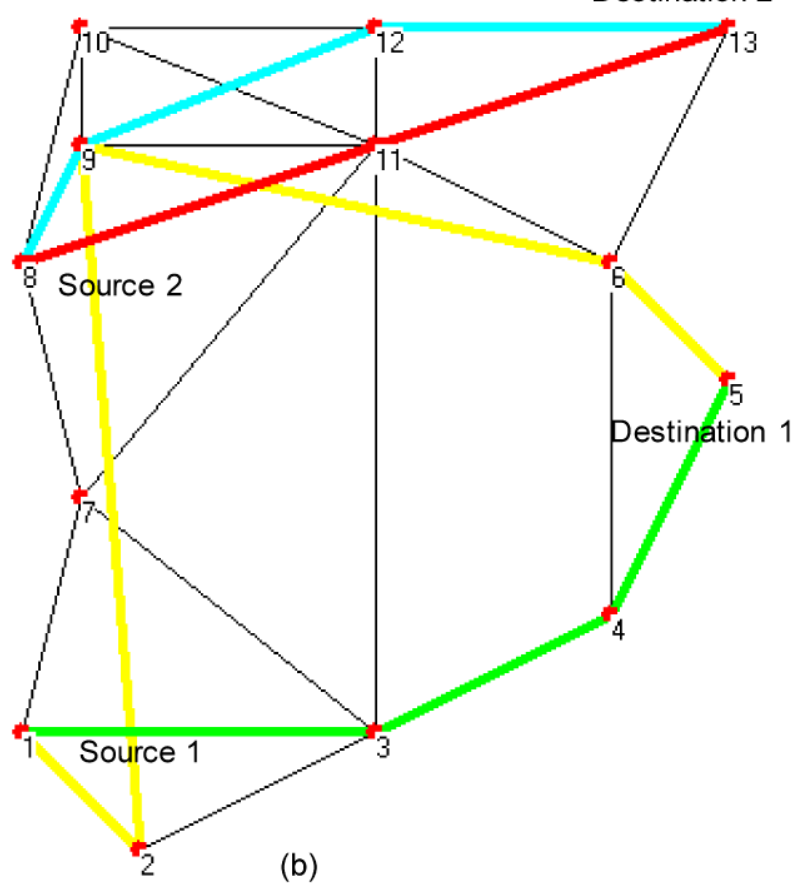

text

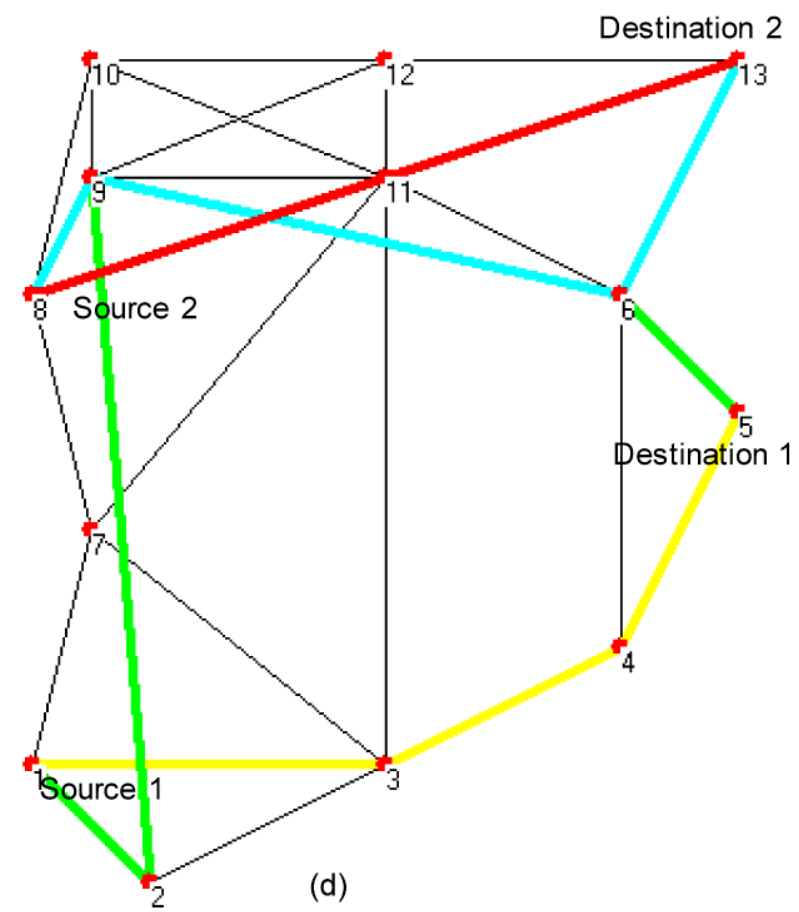

Fig. 1 Examples of Pareto solutions obtained by the proposed approach using Pareto dominance

\subsubsection{Solution improvement}

First improvement technique Let us consider the first solution given above.

$$
\begin{aligned}
& \mathrm{P} \Rightarrow 1 \begin{array}{llllllllll}
3 & 11 & 8 & 9 & 10 & 12 & 13 & 6 & 5
\end{array} \\
& \mathrm{~B} \Rightarrow 1 \begin{array}{llll}
1 & 3 & 4 & 5
\end{array}
\end{aligned}
$$

$P \Rightarrow 8 \begin{array}{llllllll}8 & 1 & 3 & 4 & 5 & 6 & 13\end{array}$

$\mathrm{B} \Rightarrow \begin{array}{lllllll}8 & 7 & 3 & 4 & 6 & 13\end{array}$

Suppose the first and fourth paths are selected (this means, primary path between node 1 and node 5 and the backup path between node 8 and node 13). A number between 1 and the length of the primary path selected (which is 10) 


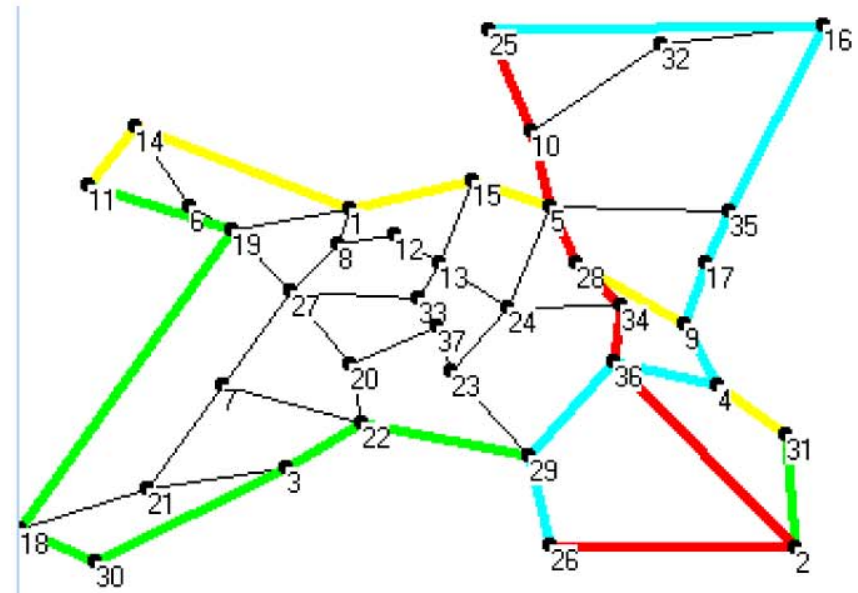

(a)

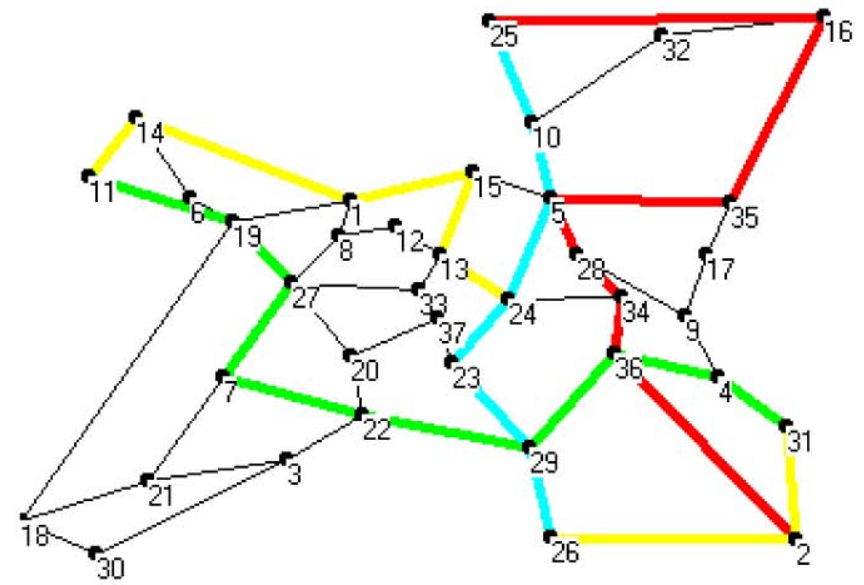

(c)

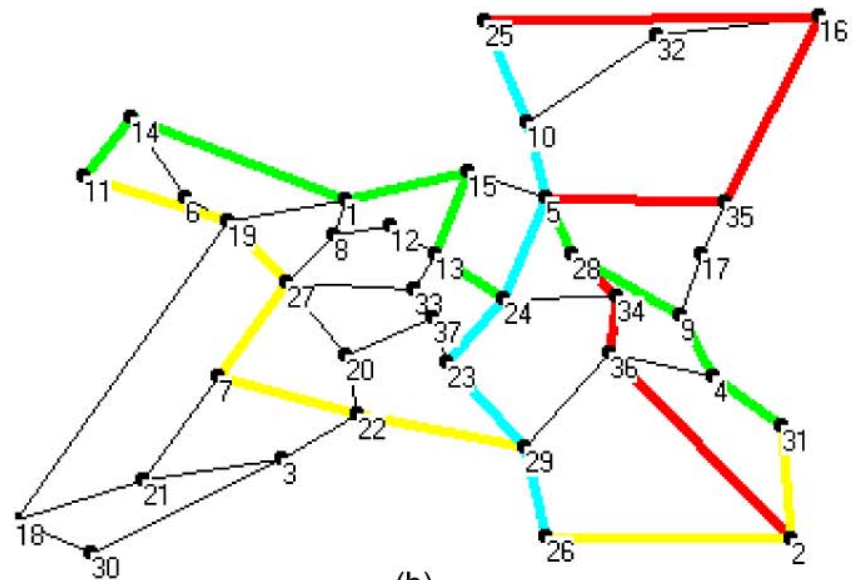

(b)

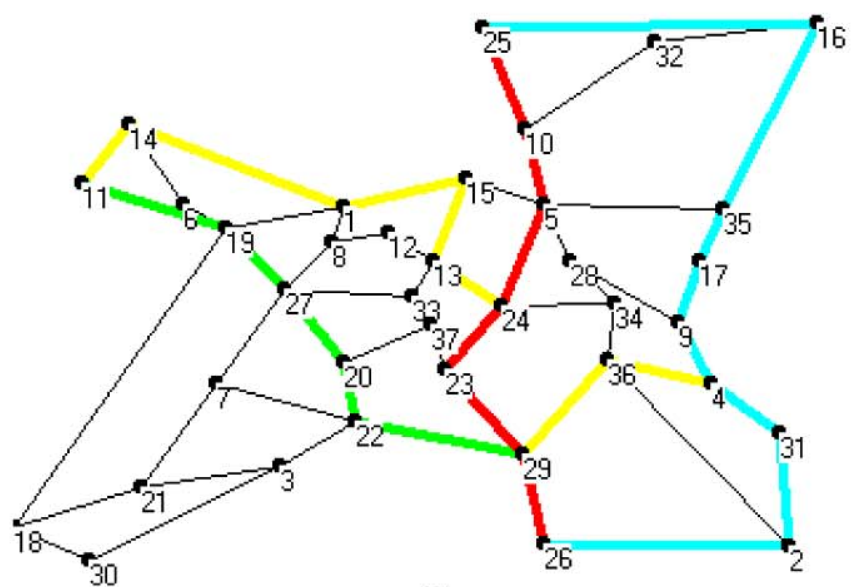

(d)

Fig. 2 Examples of solutions obtained for the Europe network benchmark. The source-destination pairs are $(11,31)$ and $(25,26)$

minus 1 is generated. Suppose number 2 is generated. This means, starting with the second position (which is node 3) this path is rebuild.

The new obtained path can be for example:

$P \Rightarrow 1311 \quad 6 \quad 5$

Same procedure is applied for the backup selected for improvement. Let the newly obtained path is:

$\mathrm{B} \Rightarrow \begin{array}{llllll}8 & 7 & 3 & 11 & 12 & 13\end{array}$

Then, the newly obtained solution is:

$$
\begin{aligned}
& P \Rightarrow 1 \quad 3 \quad 11 \quad 6 \quad 5 \\
& \mathrm{~B} \Rightarrow \begin{array}{llll}
1 & 3 & 4 & 5
\end{array} \\
& \mathrm{P} \Rightarrow 8 \begin{array}{llllllll}
8 & 1 & 3 & 4 & 5 & 6 & 13
\end{array} \\
& \mathrm{~B} \Rightarrow \begin{array}{llllll}
8 & 7 & 3 & 11 & 12 & 13
\end{array}
\end{aligned}
$$

Third improvement Consider that third and forth solutions are considered to be combined following the description of the third improvement technique. Also, consider that first backup paths are the ones which will be combined and the cutting point is at the node 3 :

$\mathrm{B} \Rightarrow \begin{array}{llllllllllll}1 & 7 & 3 & 2 & 9 & 8 & 10 & 12 & 13 & 11 & 6 & 5\end{array}$

$B \Rightarrow 1 \quad 2 \quad 3 \quad 11 \quad 6 \quad 5$

The obtained paths are:

$B \Rightarrow 1 \quad 7 \quad 3 \quad 11 \quad 6 \quad 5$

$\mathrm{B} \Rightarrow 1 \begin{array}{llllllllllll}1 & 2 & 2 & 9 & 8 & 10 & 12 & 13 & 11 & 6 & 5\end{array}$

After refining and correcting the solutions, the final paths are:

$B \Rightarrow 1 \quad 7 \quad 3 \quad 11 \quad 6 \quad 5$

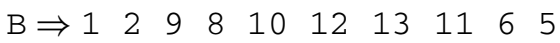

Some nondominated solutions obtained by considering the Pareto dominance relationship are depicted in Fig. 1. For the weighted sum method we considered all the objectives to have the same weights. In this situation, we obtain only 
Fig. 3 Experimental network model with 65 nodes and 108 connections

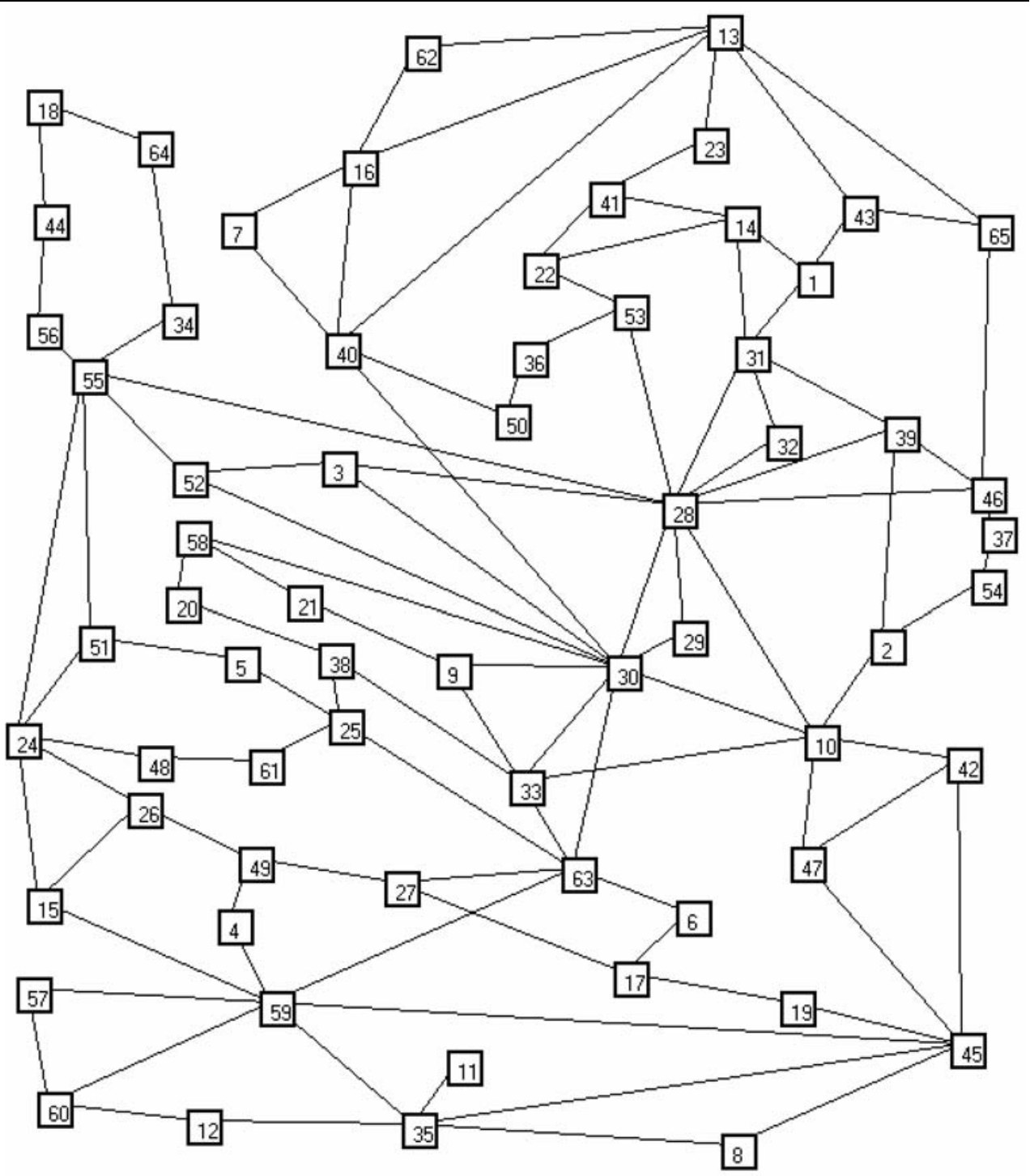

one solution. Multiple solutions may be obtained by varying the weights.

\subsection{Second example: Europe network}

We consider the Europe network benchmark, which consists of 37 nodes. The problem is to find primary and backup paths between the pairs $(11,31)$ and $(25,26)$. The cost of each existing link is set to be one.

The parameters used by the proposed method are the same as the ones used for the first example. A set of 8 feasible solutions is obtained at the end of the search process. Four of the developed solutions are depicted in Fig. 2. The value of the second objective is 0 for the obtained solutions, which means there are no common edges between a primary path and its corresponding backup.

\subsection{Third example}

Consider the network having 65 nodes and 108 connections as depicted in Fig. 3. We applied the techniques considering the following 2 cases:

- 3 sources and 3 destinations: $(24,45),(28,35),(7,46)$;

- 4 sources and 4 destinations: $(24,45),(28,35),(7,46)$, $(18,42)$.

Some nondominated solutions obtained by the multiobjective approach are depicted in Figs. 4 and 5. Four nondominated solutions for the first case and 2 solutions for the second case are presented. The solutions were picked from the whole set of nondominated solutions obtained in 30 independent runs. For a set of 10 initial solutions and 15 iterations we obtained between 1 and 5 nondominated solutions in one run. The improvement of cost objective (fitness) is depicted in Fig. 6 and the perfor- 


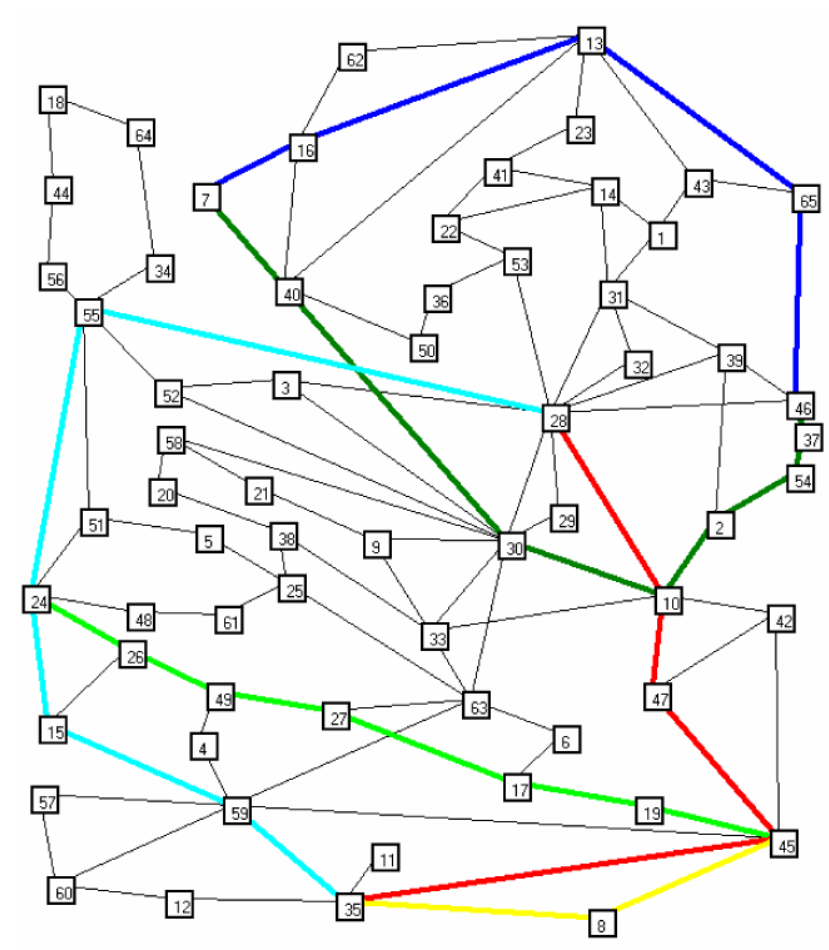

(a)

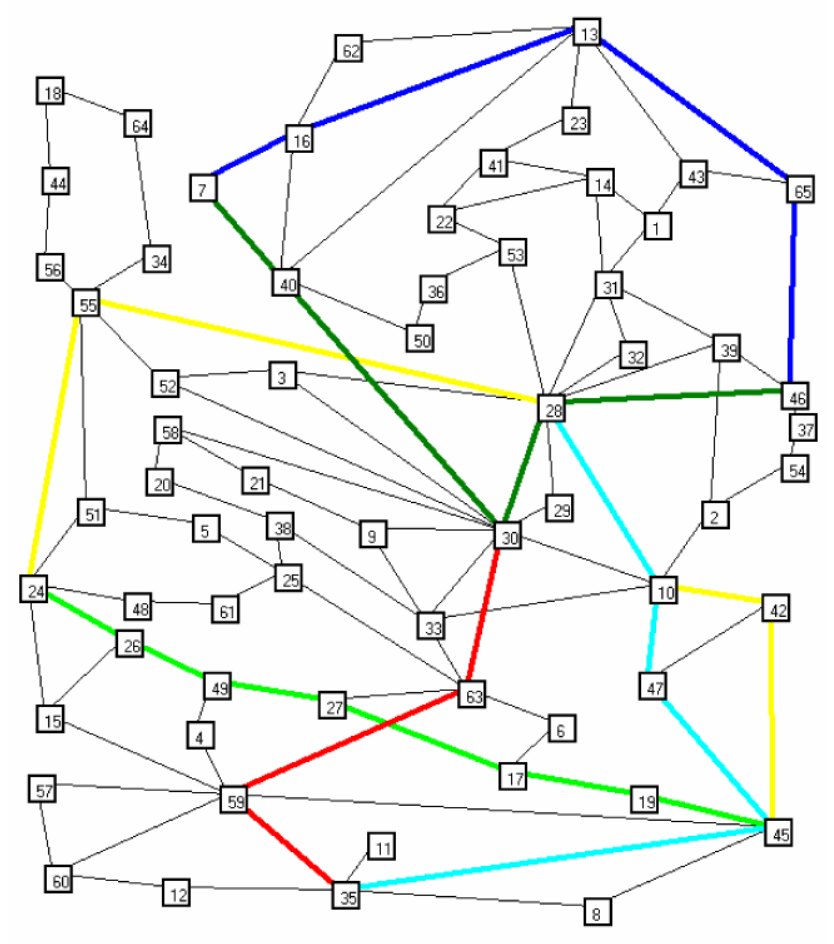

(c)

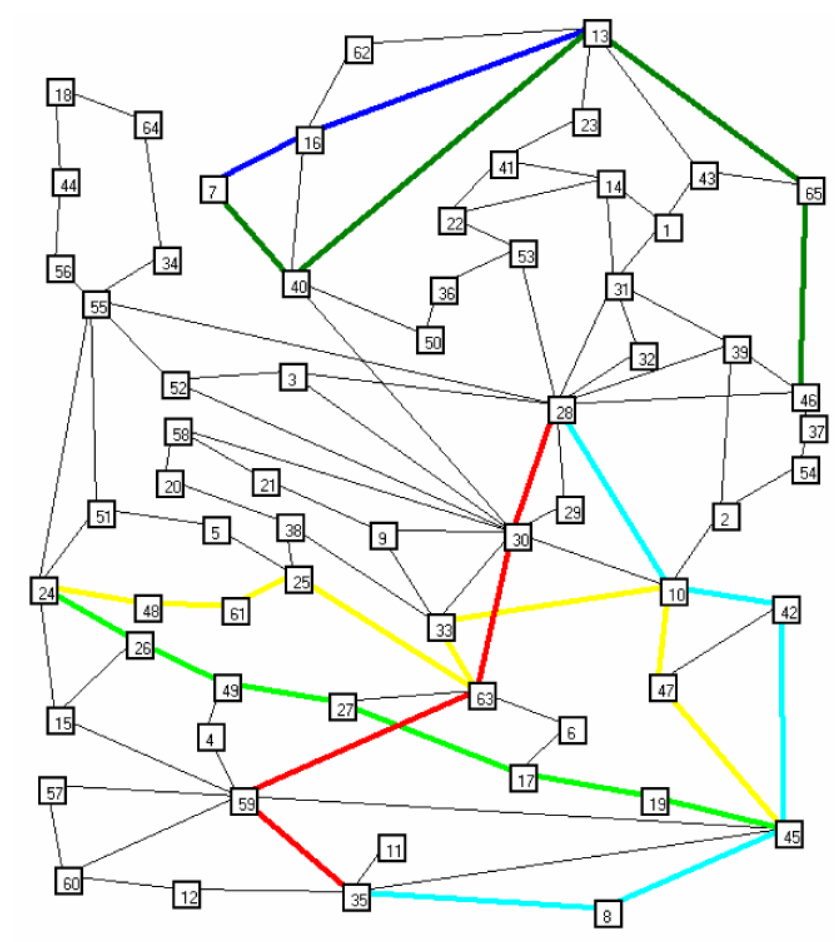

(b)

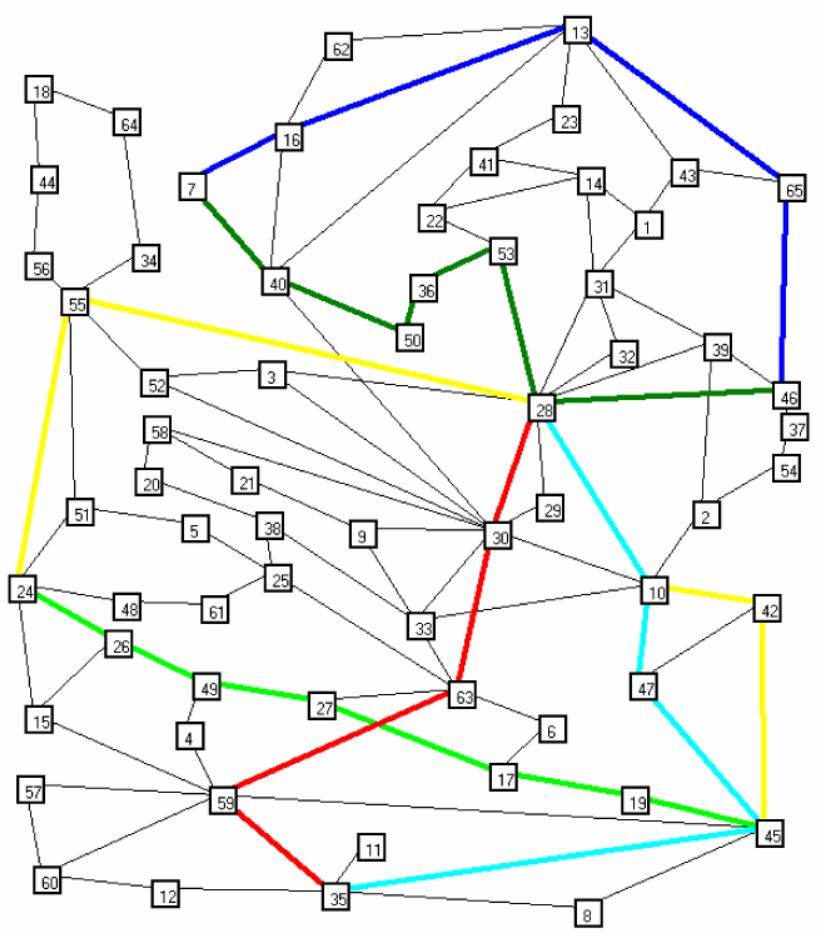

(d)

Fig. 4 (a-d) Four nondominated solutions obtained by considering Pareto dominance relationship for the third example having 3 source nodes and 3 destination nodes given by the pairs: $(24,45),(28,35),(7,46)$ 
Fig. 5 (a-b) Two

nondominated solutions obtained by considering Pareto dominance relationship for the third example having 4 source nodes and 4 destination nodes given by the pairs: $(24,45)$, $(28,35),(7,46),(18,42)$

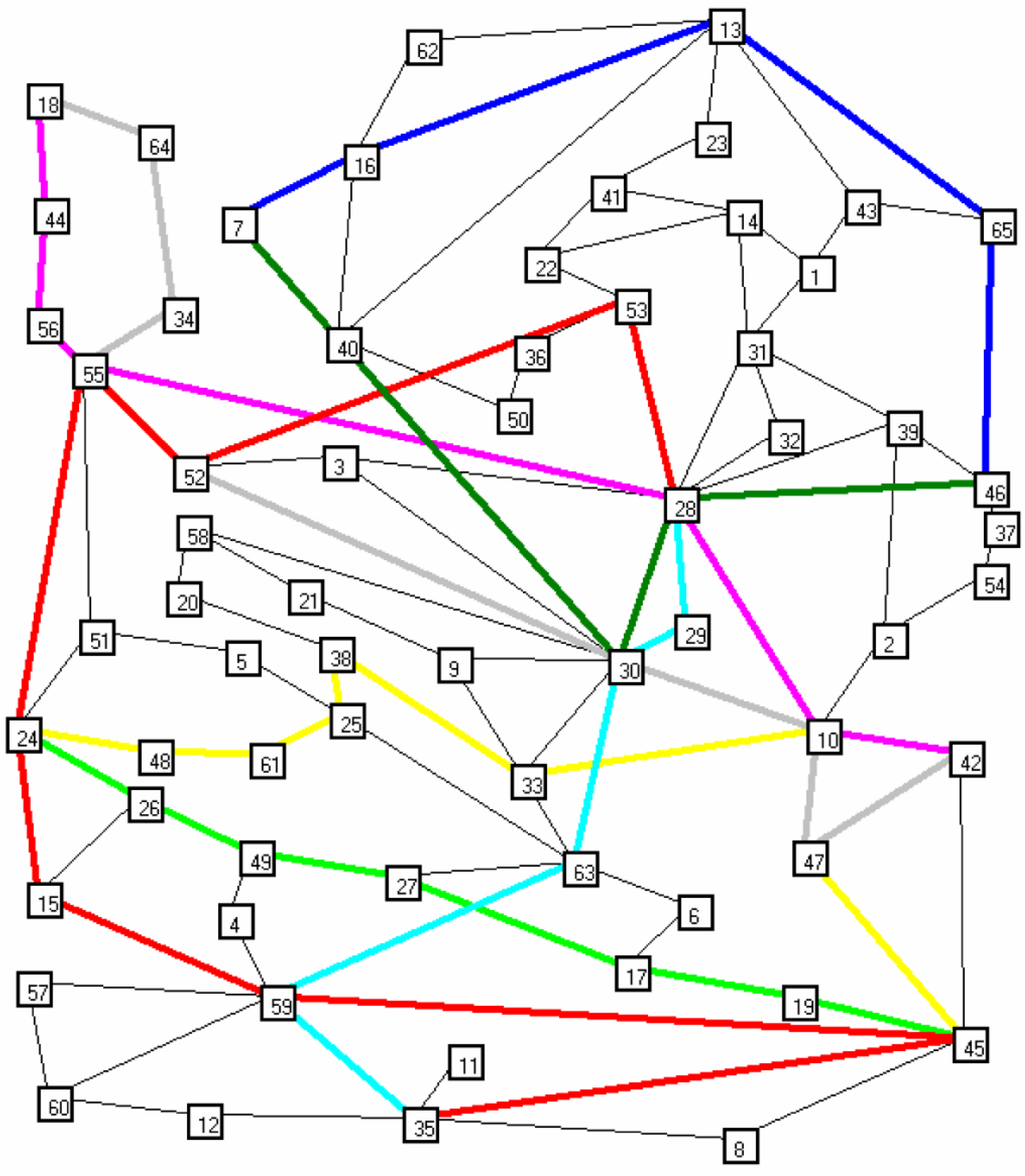

(a) mance of the objective giving the number of common edges between primary and backup paths is depicted in Fig. 7.

The evolution of the criteria considered for the single objective approach is depicted in Fig. 8. For the weighted approach, the objective weights were set to have the same value.

\section{Conclusions}

The paper proposes an algorithm dealing with multiobjectivity in resilience network. The Pareto Resilience Model (PRM) proposed herein focuses on simultaneous allocation of both primary and backup paths. Several criteria to be optimized at one time can be considered but we used only 3 objectives. Capacitated networks are considered while capacity is treated as a constrained. The performance of the proposed approach is tested for three different networks and the approach is able to detect multiple feasible solutions in a very short time (less than a second). Since the proposed model uses only few ways to improve a solution and no modality to combine two existing solutions, we propose as future work the introduction of multiple ways to generate and obtain new solutions from the existing ones, which can increases the diversity of the results at the end of the search process. It is worth to mention that an advantage of this technique is the computational time which is very less for all the considered experiments for both Pareto dominance based approach and weighted sum method (less than one second). 
Fig. 5 (Continued)

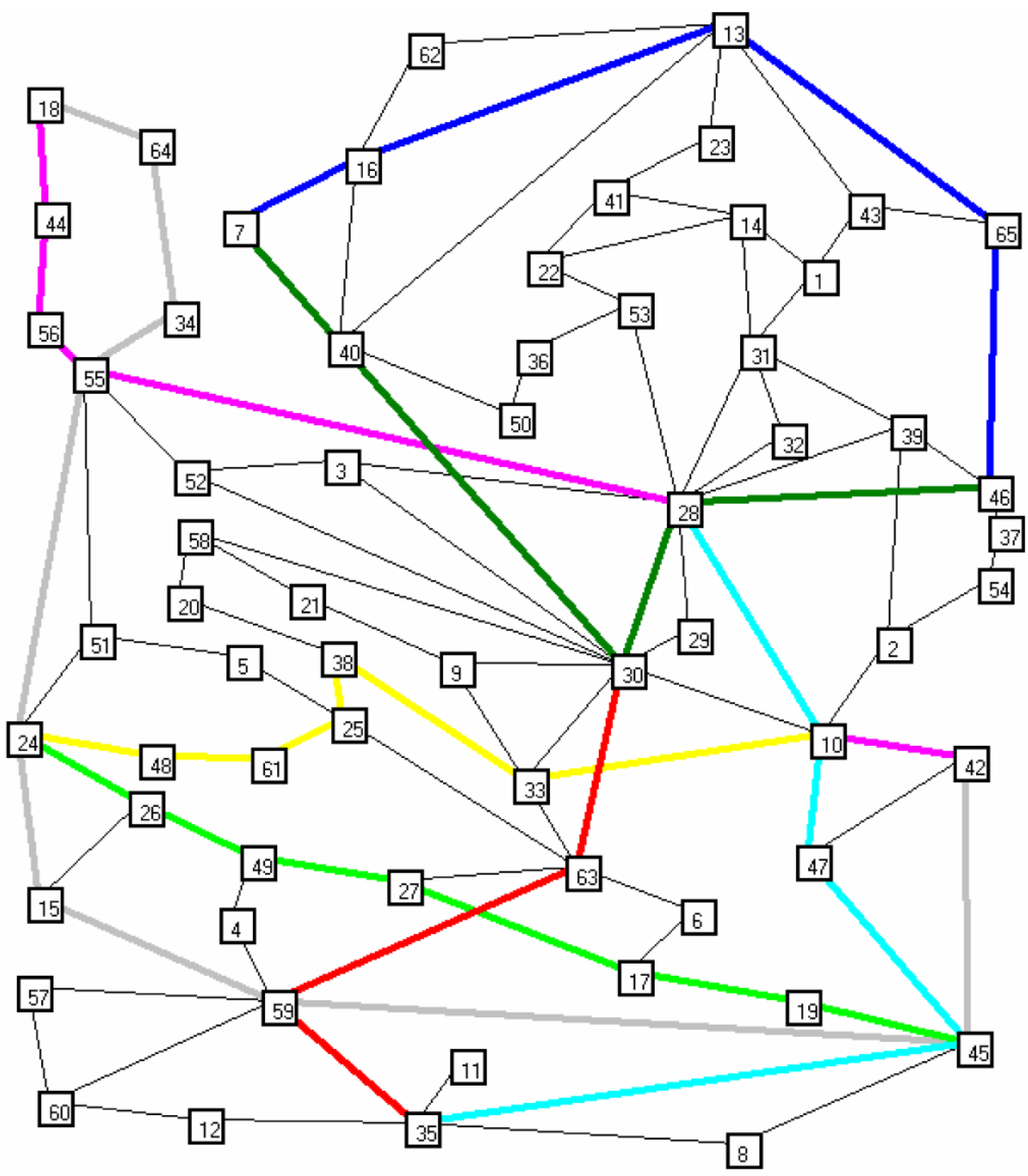

(b)

Fig. 6 Performance for the third example while considering the problem as multiobjective and using Pareto dominance

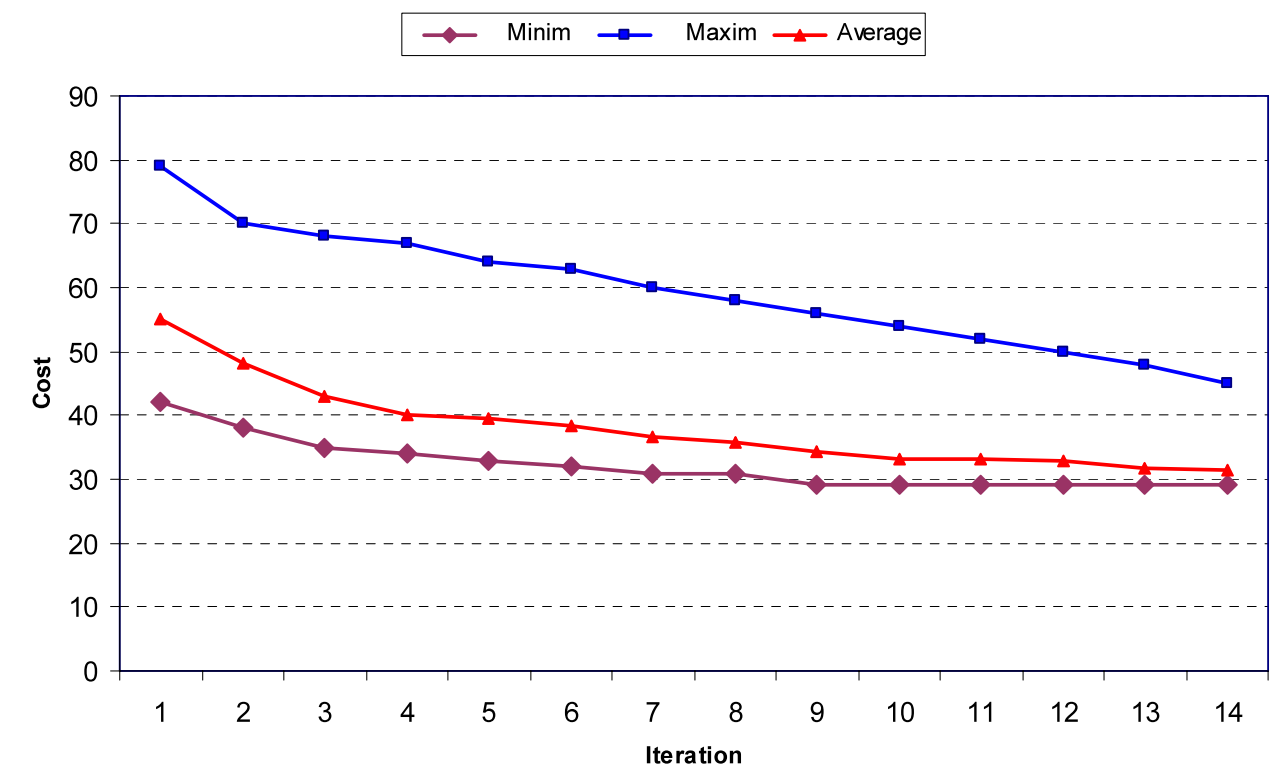


Fig. 7 Performance of the objectives cost and number of common edges for the third example while considering the problem as multiobjective and using Pareto dominance
Fig. 8 Performance using the weighted sum of the objectives for the third example (considering the problem as single objective)
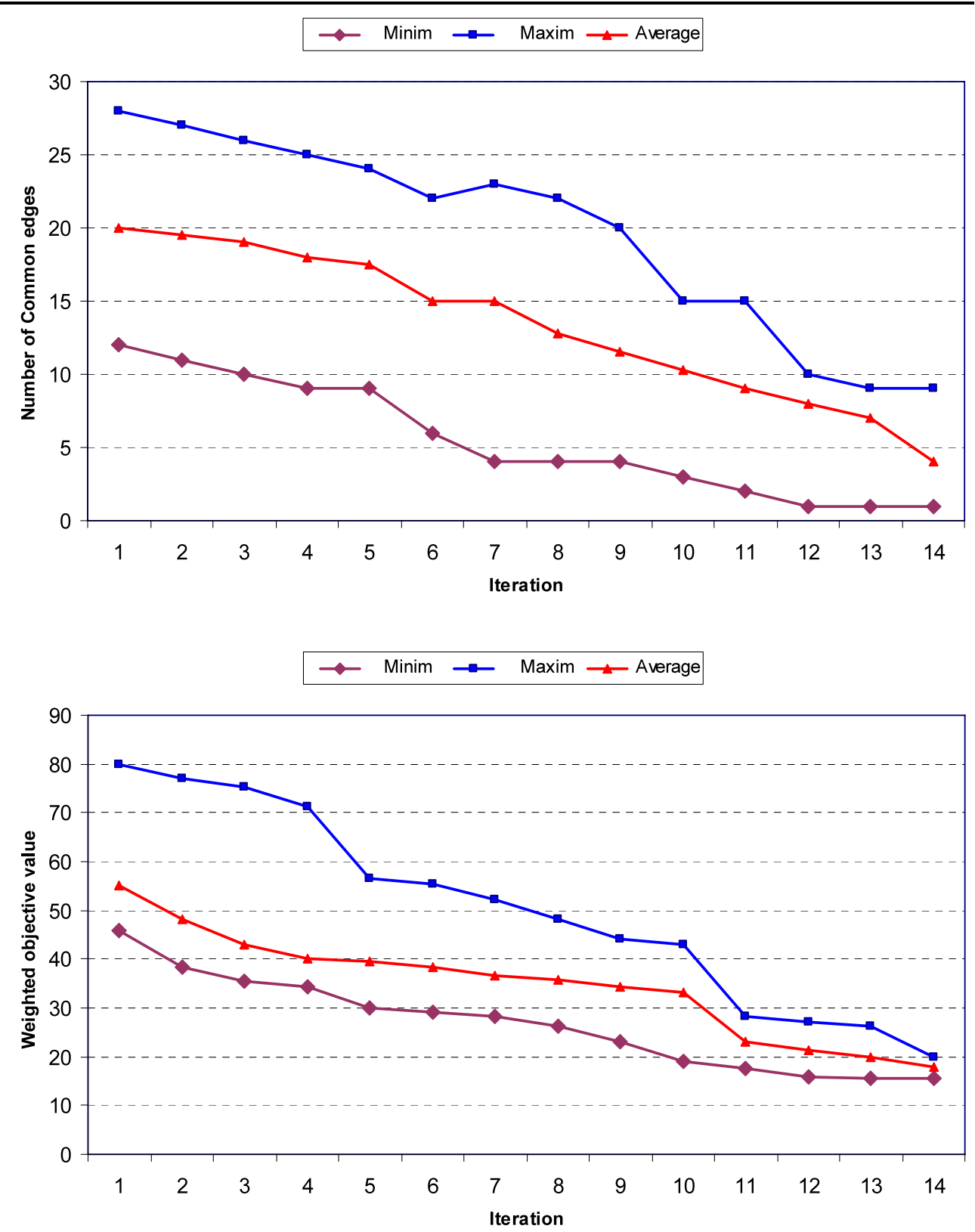

\section{References}

1. Chekuri, C., Gupta, A., Kumar, A., Naor, J., \& Raz, D. (2002). Building edge-failure resilient networks. In Proceedings of the 9 th integer programming and combinatorial optimization conference, (pp. 439-456), Cambridge, MA.

2. He, L., \& Mort, N. (2007). Hybrid genetic algorithms for telecommunications network back-up routing. BT Technology Journal, 18(4), 42-50.

3. Banarjee, N., \& Kumar, R. (2007). Multiobjective network design for realistic traffic models. In Proceedings of generic and evolutionary computation conference (GECCO'07), (pp. 1904-1911), London, UK.

4. Bremler-Barr, A., Afek, Y., Cohen, E., Kaplan, H., \& Merritt, M. (2001). Restoration by path con-catenation: fast recovery of MPLS paths. In Proceedings of the ACM PODC '01.
5. Balakrishnan, A., Magnanti, T., Sokol, J., \& Wang, Y. (2002). Spare-capacity assignment for line restoration using a singlefacility type. Operations Research, 50(4), 617-635.

6. Balakrishnan, A., Magnanti, T., Sokol, J., \& Wang, Y. (2001). Telecommunication link restoration planning with multiple facility types. Annals of Operations Research, 106(1-4), 127-154.

7. Bienstock, D., \& Muratore, G. (2001). Strong inequalities for capacitated survivable network design problems. Mathematical Programming, 89, 127-147.

8. Brightwell, G., Oriolo, G., \& Shepherd, F. B. (2001). Reserving resilient capacity in a network. SIAM Journal of Discrete Mathematics, 14(4), 524-539.

9. Brightwell, G., Oriolo, G., \& Shepherd, F. B. (2003). Reserving resilient capacity with upper bound constraints. Networks, 41(2), 87-96. 
10. Dahl, G., \& Stoer, M. (1998). A cutting plane algorithm for multicommodity survivable network design problems. INFORMS Journal on Computing, 10, 1-11.

11. Yeh, W. C. (2007). An interactive augmented max-min MCS-RSM method for the multi-objective network reliability problem. International Journal of Systems Science, 38(2), 87-99.

12. Grosan, C., Abraham, A., \& Helvik, B. (2008). Building multiobjective resilient networks. In Tenth international conference on computer modeling and simulation Eurosim/UKSim (pp. 204209). Cambridge: IEEE Computer Society Press.

13. Grover, W. D. (2003). Mesh-based survivable transport networks: options and strategies for optical, MPLS, SONET and ATM networking. New York: Prentice Hall.

14. Pioro, M., \& Medhi, D. (2004). Routing, flow and capacity design in communication and computer networks. San Mateo: Morgan Kaufmann (ISBN 0125571895).

15. Vasseur, J.-P., Pickavet, M., \& Demeester, P. (2004). The Morgan Kaufmann series in networking. Network recovery: protection and restoration of optical, SONET-SDH, IP, and MPLS. San Mateo: Morgan Kaufmann.

16. Kim, I. Y., \& deWeck, O. L. (2005). Adaptive weighted-sum method for bi-objective optimization: Pareto front generation. Structural and Multidisciplinary Optimization, 29, 149-158.

17. Zadeh, L. (1963). Optimality and non-scalar-valued performance criteria. IEEE Transaction on Automation Control, 8, 59-60.

18. Stadler, W. (1979). A survey of multicriteria optimization, or the vector maximum problem. Journal of Optimization Theory and Application, 29, 1-52.

19. Steuer, R. E. (1986). Multiple criteria optimization: theory, computation and application. New York: Wiley.

20. De Vleeschouwer, C., \& Frossard, P. (2008). Loss-resilient window-based congestion control. Computer Networks, 52(7), 1473-1491.

21. Wouhaybi, R. H., \& Campbell, A. T. (2008). Building resilient low-diameter peer-to-peer topologies. Computer Networks, 52(5), 1019-1039.

22. Antonino, A., Bianco, A., Bianciotto, A., De Feo, V., Finochietto, J. M., Gaudino, R., \& Neri, F. (2008). WONDER: a resilient WDM packet network for metro applications. Optical Switching and Networking, 5(1), 19-28.

23. Deng, Y. (2008). RISC: A resilient interconnection network for scalable cluster storage systems. Journal of Systems Architecture, 54(1-2), 70-80.

24. Konak, A., \& Bartolacci, M. R. (2007). Designing survivable resilient networks: a stochastic hybrid genetic algorithm approach. Omega, 35(6), 645-658.

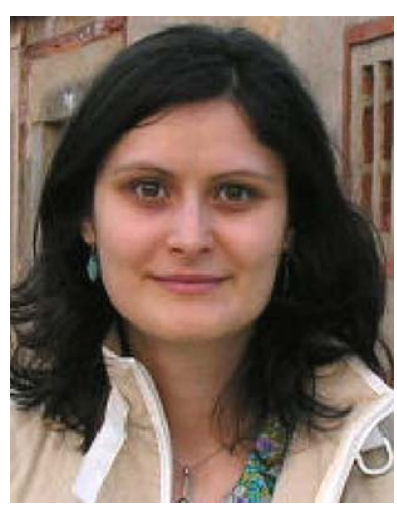

Crina Grosan received her MS degree in Mathematics and $\mathrm{PhD}$ degree in Computer Science from Babes-Bolyai University, ClujNapoca, Romania in 2005. She is a Lecturer of Artificial Intelligence at Babes-Bolyai University. Her research focuses on different global optimization techniques and applications. She has been researching such topics as multiobjective optimization, global optimization, operations research, numerical analysis, computational intelligence and swarm intelligence. She has published over 100 research articles in peer reviewed international journals, book chapters and conference proceedings. She is co-author of two books on programming languages and is also a co-editor of four volumes titled Stigmergic Optimization, Swarm Intelligence in Data Mining, Hybrid Evolutionary Algorithms and Engineering Evolutionary Intelligent Systems, published by Springer Verlag, Germany. She guest edited a special issue on Soft Computing for Modeling and Simulation for the International Journal of Simulation Systems, Science \& Technology, published by the UK Simulation Society. She is the managing editor of International Journal of Computational Intelligence Research and also serves the editorial board of few other Journals. She co-founded the Evolutionary Multiobjective Optimization: Design and Applications (EMODA) International workshop series in 2005.

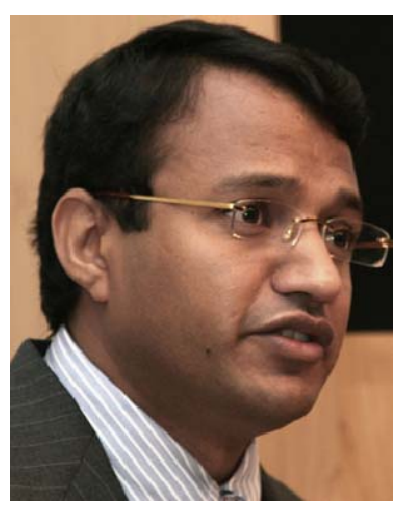

Ajith Abraham research and development experience includes over 18 years in the Industry and Academia spanning different continents in Australia, America, Asia and Europe. He works in a multi-disciplinary environment involving computational intelligence, network security, sensor networks, e-commerce, Web intelligence, Web services, computational grids, data mining and applied to various real world problems. He has authored/coauthored over 400 refereed journal/conference papers and book chapters and some of the works have also won best paper awards at international conferences and also received several citations. Some of the articles are available in the ScienceDirect Top 25 hottest articles. He has given more than 20 plenary lectures and conference tutorials in these areas.

Currently, he is working with the Norwegian University of Science and Technology, Norway. Before joining NTNU, he was working under the Institute for Information Technology Advancement (IITA) Professorship Program funded by the South Korean Government. He was a Researcher at Rovira i Virgili University, Spain during 2005-2006. He also holds an Adjunct Professor appointment in Jinan University, China and Dalian Maritime University, China. He has held academic appointments in Monash University, Australia; Oklahoma State University, USA; Chung-Ang University, Seoul and Yonsei University, Seoul. Before turning into a full time academic, he was working with three International companies: Keppel Engineering, Singapore, Hyundai Engineering, Korea and Ashok Leyland Ltd, India where he was involved in different industrial research and development projects for nearly 8 years. He received Ph.D. degree in Computer Science from Monash University, Australia and a Master of Science degree from Nanyang Technological University, Singapore.

He serves the editorial board of over 30 reputed International journals and has also guest edited 28 special issues on various topics. He is actively involved in the Hybrid Intelligent Systems (HIS); Intelligent Systems Design and Applications (ISDA) and Information Assurance and Security (IAS) series of International conferences. He is a Senior Member of IEEE (USA), IEEE Computer Society (USA), IET (UK), IEAust (Australia) etc.

In 2008, he is the General Chair/Co-chair of Tenth International Conference on Computer Modeling and Simulation, (UKSIM'08), Cambridge, UK; Second Asia International Conference on Modeling \& Simulation (AMS'08), Kuala lumpur, Malaysia; Eight International Conference on Intelligent Systems Design and Applications (ISDA'08), Kaohsiung, Taiwan; Fourth International Symposium on Information Assurance and Security (IAS'08), Naples, Italy; 2nd European Symposium on Computer Modeling and Simulation, (EMS'07), Liverpool, UK; Eighth International Conference on Hybrid Intelligent Systems (HIS'08), Barcelona, Spain; Fifth International Conference on Soft Computing as Transdisciplinary Science and Technology (CSTST'08), Paris, France Program Chair/Co-chair of Third Interna- 
tional Conference on Digital Information Management (ICDIM'08), London, UK; 7th Computer Information Systems and Industrial Management Applications (CISIM'08), Ostrava, Czech Republic; Second European Conference on Data Mining (ECDM'08), Amsterdam, Netherlands and the Tutorial Chair of 2008 IEEE/WIC/ACM International Joint Conference on Web Intelligence and Intelligent Agent Technology (WI-IAT'08), Sydney, Australia More information at: http://www.softcomputing.net.

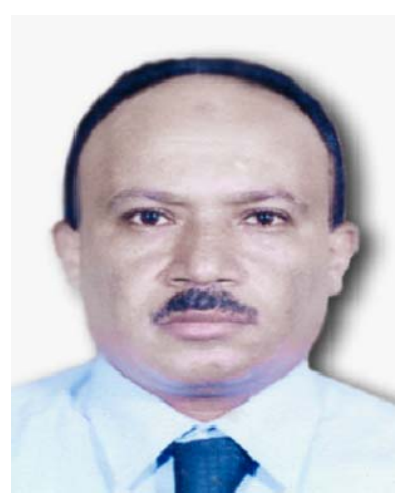

Aboul Ella Hassainen received his BSc with honours in 1986 and MSc degree in 1993, both from Ain Shams University, Faculty of Science, Pure Mathematics and Computer Science Department, Cairo, Egypt. In 1998, he received his doctoral degree from the Department of Computer Science, Graduate School of Science and Engineering, Tokyo Institute of Technology, Japan. He is an Associate Professor at Cairo University, Faculty of Computer and Information, IT Department. He has authored/coauthored over 80 research publications in peer-reviewed reputed journals and conference proceedings. He has served as the program committee member of various international conferences and reviewer for various international journals. Since 2004, he is actively involved as technical committee in the International Association of Science and Technology for Development (IASTED) for Image Processing and Signal Processing. He has received the excellence younger researcher award from Kuwait University for the academic year 2003/2004. He has guest edited many special issues for international scientific journals. He has directed many funded research projects. Dr. Abo was a member of the Interim Advisory Board committee of the International Rough Set Society. He is the editor and co-editor for more than six books in the area of rough computing, computational intelligence, and E-commerce.His research interests include, rough set theory, wavelet theory, X-ray Mammogram analysis, medical image analysis, fuzzy image processing and multimedia data mining. 